\title{
Microfluidic Sandwich ELISA Utilizing a Concave Microchannel Surface Generated by Nanosecond Laser Ablation
}

\author{
Yusuke Fuchiwaki ${ }^{1,4,}{ }^{*}$, Masato Tanaka ${ }^{1}$, Kaori Abe ${ }^{2,3}$, \\ Masatoshi Kataoka ${ }^{2}$ and Toshihiko Ooie ${ }^{1}$ \\ 'Biodevice Research Group, Health Research Institute, National Institute of Advanced Industrial \\ Science and Technology (AIST), 2217-14, Hayashi-cho, Takamatsu, Kagawa 761-0395, Japan \\ ${ }^{2}$ Biomarker Analysis Research Group, Health Research Institute, \\ National Institute of Advanced Industrial Science and Technology (AIST), \\ 2217-14, Hayashi-cho, Takamatsu, Kagawa 761-0395, Japan \\ ${ }^{3}$ Clinical Research Center for Diabetes, Tokushima University Hospital, \\ 2-50-1, Kuramoto-cho, Tokushima 770-8503, Japan \\ ${ }^{4}$ CEA-LETI, Minatec Campus, 17 rue des Martyrs, 38054 Grenoble Cedex 9, France
}

(Received January 29, 2015; accepted April 21, 2015)

Key words: enzyme-linked immunosorbent assay, laser ablation, concave, point-of-care testing, poly(methyl methacrylate)

A practical point-of-care sandwich enzyme-linked immunosorbent assay (ELISA) microchip remains elusive. A concave area on the undersurface of a microchannel was formed by nanosecond pulse laser ablation and its utility for a microfluidic system based on capillary-driven flow supporting such a microchip was determined. Ablation using a $193 \mathrm{~nm}$ wavelength, $1.76 \mathrm{~J} \cdot \mathrm{cm}^{-2} \mathrm{UV}$ laser modified the surface of poly(methyl methacrylate), providing a surface amenable for antibody immobilization. A concave microchannel $10 \mu \mathrm{m}$ deep and $260 \mu \mathrm{m}$ in diameter allowed both successful antibody microdeposition and complete reagent replacement required for a practical ELISA microchip. Quantitative analysis of concentrations of procollagen-type I carboxyterminal propeptide (PICP) using this microchannel showed a linear relationship at PICP concentrations between $0-600 \mathrm{ng} \cdot \mathrm{ml}^{-1}$, which is adequate for clinical estimation of PICP concentrations in the blood.

\section{Introduction}

Point-of-care testing (POCT) devices are becoming increasingly sophisticated and are finding widespread application in the field of medical diagnosis. Most POCT devices employ immunoassay methods; specifically, the enzyme-linked immunosorbent assay

*Corresponding author: e-mail: yu-fuchiwaki@aist.go.jp 
(ELISA) is an established standard method for detecting several disease biomarkers, such as inflammatory cytokines, in blood samples. Conventional sandwich ELISA systems are based on disposable 96-well microtitration plates. This format requires timeconsuming incubations and multiple washing steps, which limit its throughput and utility for rapid diagnosis. Miniaturization of conventional ELISA assays into POCT devices offers many advantages in terms of simplicity, portability, and automation, as well as being easy to operate, providing rapid results, and requiring small sample sizes. To date, several types of front-line microchips have been developed for chemical and biological analyses. Antibodies adsorbed onto beads packed in microchannels are commonly used for sandwich ELISAs. ${ }^{(1-7)}$ These immunoassay systems use elaborate microscale dam-like structures to retain the microbeads in the microchannels during solution exchange steps required by the immunoassay process. Inkjet printing has been used to microdeposit antibodies onto a substrate to produce practical and reproducible POCT devices $^{(8-11)}$ and allows picoliter quantities of liquid to be microdeposited in a single process with drop-on-demand control.

We recently fabricated an immunoassay microchip device for ELISA using a piezoelectric inkjet printing system manufactured by Cluster Technology Co., Ltd. (Osaka, Japan). ${ }^{(9-11)}$ This microchip was used to construct a sandwich ELISA for the quantitative analysis of biomarkers in blood. The piezoelectric inkjet printing system was used to deposit and fix primary antibody onto a chemically-modified plastic microchannel surface. However, despite these developments, it remains difficult to immobilize antibodies in specific locations with sufficiently high reproducibility.

Recently, advances in laser ablation technology now allow highly precise, fast, and gentle modification of plastic surfaces. ${ }^{(12-17)}$ Pulse laser ablation is an attractive approach for topographically and chemically modifying surfaces. Polymer cohesion energy produced by a UV nanosecond pulse laser breaks predominantly inter-chain bonds. ${ }^{(12,13)}$ However, the precise mechanism underlying laser ablation of poly(methyl methacrylate) (PMMA) remains to be elucidated due to its complexity; it cannot currently be described by a single photochemical or mathematical model. Nonetheless, various laser ablation technologies based on surface modification have been proposed for protein immobilization ${ }^{(18,19)}$ and wettability modification, ${ }^{(14,15)}$ suggesting that surfaces can be modified by laser ablation for antibody immobilization. We previously described that UV laser irradiation of a plastic surface enhanced the affinity of the surface for antibodies. ${ }^{(18,20)}$ Surprisingly, little or no attention has been paid to using nanosecond pulse laser ablation for surface modification, even though this technique allows efficient antibody immobilization and provides the basis for a practical immunoassay chip. The ablation of PMMA using $193 \mathrm{~nm}$ laser light with an intensity of $1.76 \mathrm{~J} \cdot \mathrm{cm}^{-2}$ induces morphological changes in the remaining substrate due to strong absorption of laser energy. Optical analyses showed that PMMA remains essentially transparent after laser ablation at $193 \mathrm{~nm}$, whereas other plastic polymers such as cyclic olefin copolymer (COC) and polystyrene (PS) darken significantly. ${ }^{(18,21,22)}$ Consequently, we used laser ablation at $193 \mathrm{~nm}$ and $1.76 \mathrm{~J} \cdot \mathrm{cm}^{-2}$ to modify the surface of PMMA and demonstrated that many antibodies can be immobilized onto PMMA microchannel surfaces. ${ }^{(18,20)}$ 
A practical sandwich micro ELISA system for POCT applications requires highly specific and localized antibody immobilization compatible with highly reproducible solution replacement. Inkjet printing can precisely pattern picoliter quantities of liquid, but the high concentration of primary antibody required for sensitive ELISA destabilizes the ejection spray from the inkjet printing head due to the high viscosity of the droplet caused by the high antibody concentration.

We here describe the basis of a next generation practical POCT immunoassay microfluidic chip. A concave microchannel surface was generated to allow reproducible antibody microdeposition at specific locations, and nanosecond pulse laser ablation of the concave undersurface enhanced antibody immobilization. Comparison of several microchannel surfaces with different undersurfaces demonstrated the feasibility of a practical sandwich ELISA system.

There is no previous report of a microfluidic sandwich ELISA system utilizing a concave microchannel surface generated by nanosecond laser ablation. Also, this study could offer the first step toward a new benchmark for a fully mechatronic and largescale quality antibody immobilization system for industrial-scale adsorption. Hence, this study may provide an innovative technology for a mechatronic antibody immobilizationbased capillary flow-immunoassay chip.

\section{Materials and Methods}

\subsection{Reagents}

The sandwich ELISA microchip used the monoclonal anti-procollagen type I carboxyterminal propeptide (PICP) antibodies provided in the procollagen type I C-peptide (PIP) EIA Kit (Takara Bio Inc., Shiga, Japan). A piezoelectric inkjet printing system (Cluster Technology Co., Ltd., Osaka, Japan) was used to deposit primary antibody (Clone PC87, Takara Bio Inc.) in spotting buffer on the surface of the microchannel.

\subsection{ELISA on microchip}

The PMMA microchip was fabricated by injection molding with a nickel mold. The channels were straight, $300 \mu \mathrm{m}$ wide, and $100 \mu \mathrm{m}$ deep [Fig. 1(a)]. Capillary-driven flow in microchannels allows low volume, fast reaction, and simple operation of our previously reported microfluidic immunoassay system, providing a rapid and accurate screening methodology [Fig. 1(b)]. ${ }^{(9-11,18,20)}$ In this study, we optimized the immunoassay performance of this microfluidic system. Samples and reagents were pipetted into the sample port of the microchips, and the microchannels were filled by capillary-driven flow. The microchannels were emptied using a syringe between immunoassay steps.

To investigate the effects of microfluidic chip responses on PICP concentration, the concentration of PICP was quantitatively analyzed using $0,150,300$, and $600 \mathrm{ng} \cdot \mathrm{ml}^{-1}$ PICP solutions. Three spots of primary antibody were deposited onto separate concave surfaces in the microchannel, then different concentrations of PICP solution and peroxidase-labeled second antibody were infused into each microchannel.

The microchip was incubated for $4 \mathrm{~h}$ at room temperature, then the surface was bonded with a film of PMMA (47 $\mu \mathrm{m}$ thick) using a 33- $\mu \mathrm{m}$-thick layer of glue (Toyo Ink 


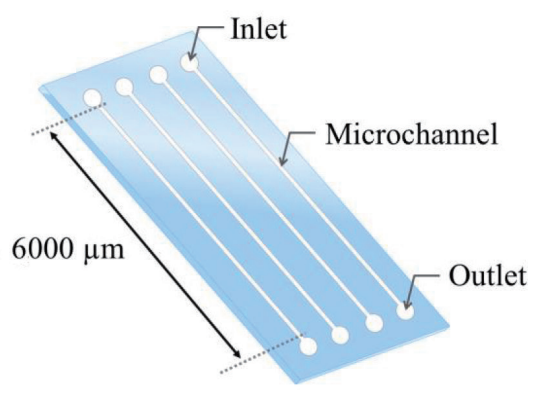

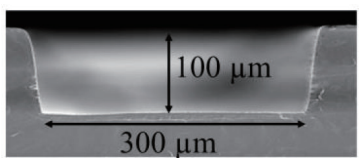

Cross-sectional view of microchannel

(a)

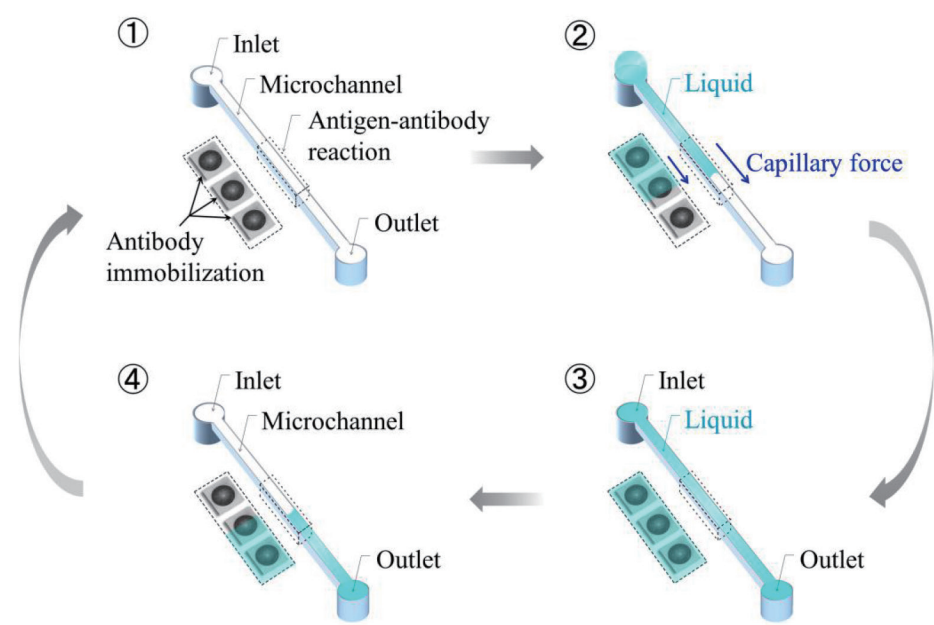

(b)

Fig. 1. (Color online) Schematic view of the microchip and the ELISA-based process using a microchip: (a) image of the microchip and photo of a cross-sectional view of the microchannel, and (b) schematic showing the procedure for fabricating a microchannel for a capillary-driven-flow immunoassay process.

Manufacturing. Co., Ltd., Tokyo, Japan). Blocking solution and washing solution were purchased from Sumitomo Bakelite Co. Ltd., Tokyo, Japan. For detection, a mixture of $2.0 \mu \mathrm{l}$ peroxidase labeled second anti-PICP antibody (Clone PC5-5, Takara Bio Inc.) and $0.4 \mu \mathrm{l}$ of the PICP serum sample was infused into each microchannel, followed by incubation at room temperature for $30 \mathrm{~min}$. After washing the microchannel, $2.0 \mu \mathrm{l}$ SuperSignal West Femto Chemiluminescent Substrate (Thermo Scientific, Rockford, IL), a chemiluminescent substrate for detecting peroxidase activity, was infused into the microchannels, and then the chip was incubated for $5 \mathrm{~min}$ at room temperature. The integrated luminescence intensity of each antibody deposition was measured using an ImageQuant LAS4000 (GE Healthcare UK Ltd., Buckinghamshire, UK), which has a resolution of up to $11 \mu \mathrm{m}$, and the data were analyzed using ImageQuant TL software (GE Healthcare UK Ltd.). 


\subsection{Equipment and modification}

A nanosecond pulse laser (LPX-305i, Coherent Inc.) was used with the following settings: pulse energy, $500 \mathrm{~mJ}$; power, $25 \mathrm{~W}$; repetition rate, $50 \mathrm{pps}$; pulse width, $14 \mathrm{~ns}$. The system was computer controlled, including the movement of the XYZ stages for translating the sample under the focused laser spot. A video microscope system allowed accurate positioning and in situ monitoring of the laser ablation process. A power monitor was used to adjust optical attenuation, reducing or increasing the power of the optical beam.

The printing head (PulseInjector ${ }^{\circledR}$ : Cluster Technology Co., Ltd., Osaka, Japan) was a piezo-driven drop-on-demand plastic head with an epoxy resin composite as the structural material; it exhibited excellent chemical resistance similar to plastics and exhibited very low biomolecular adsorption compared with glasses. Combining the PulseInjector ${ }^{\circledR}$ with a dedicated driving unit (WaveBuilder ${ }^{\circledR}$ : Cluster Technology Co., Ltd., Osaka, Japan) allowed easy adjustment of the ejection drive waveform, the ejection rate, and the driving voltage, enabling picoliter droplets to be ejected. Three types of drive waveform $(\mathrm{A}, \mathrm{B}, \mathrm{C})$ are available in a user-friendly function. Derivative waveform $\mathrm{C}$ provided stable ejection, and the droplets were ejected at a frequency of $1 \mathrm{kHz}$ and a jetting voltage of $10 \mathrm{~V}$. The volume of a single discharged droplet of primary antibody was $65 \mathrm{pl}$, and 200 droplets were discharged onto the surface of the microchannel to provide the optimal volume of the primary antibody. The PulseInjector ${ }^{\circledR}$ used a $25-\mu \mathrm{m}-$ diameter ejection hole.

\section{Results and Discussion}

\subsection{Stable ejection onto a specific site on the microchannel undersurface}

Droplet trajectory and impact were monitored to ensure ejection stability and deposition from the inkjet head before depositing primary antibody solution on the microchannel surface. The PulseInjector ${ }^{\circledR}$ closely contacted the microchannel during antibody deposition because of the flattened surface surrounding the ejection hole [Fig. 2(a)]. A high-viscosity liquid such as a solution with a high concentration of primary antibody destabilizes ejection from the inkjet head [Fig. 2(b)], causing droplets to adhere to the wall of the ejection hole due to surface tension, negatively impacting the uniformity of the printed antibody pattern. This was addressed by forming a circular concave surface (diameter: $260 \mu \mathrm{m}$ ) on a microchannel and comparing the uniformity of the antibody printed pattern [Figs. 2(c) and 2(d)]. Three spots were prepared on each channel surface, and 12 spots on 4 channels were prepared in total. Uniform areas were formed in the concave shapes and non-uniform areas were formed outside the concave areas; the printing area and relative standard deviations (RSDs) were $5.97 \mu \mathrm{m}$ and $2.2 \%$ within the concave shape and $4.97 \mu \mathrm{m}$ and $35.4 \%$ outside the concave area. Therefore, the circular concave shape was demonstrated to be effective for uniform printing of antibody patterns on a microchannel surface.

The microchannel used in this study operates on the principle of capillary-driven flow that mediates the transport of reagents between immunoassay steps. The concave surface in the microchannel is a negative structure from the standpoint of the complete 


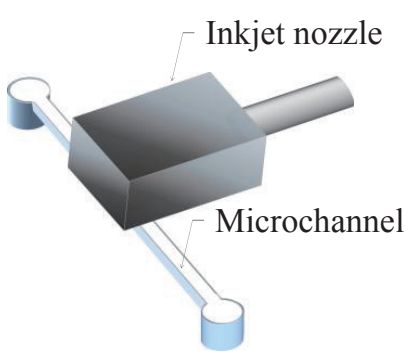

(a)
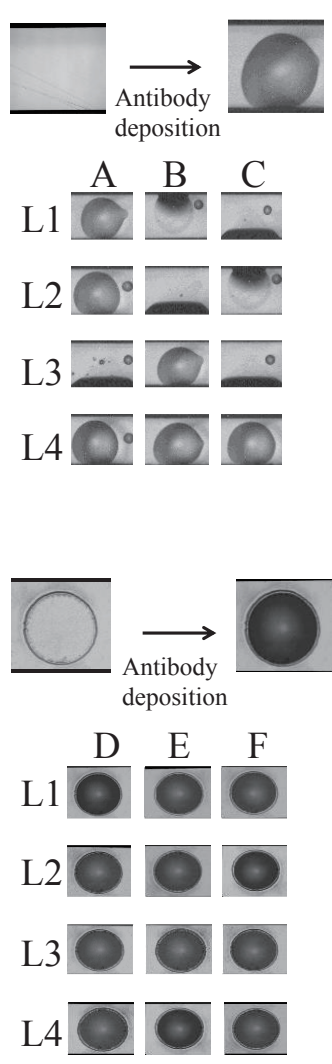

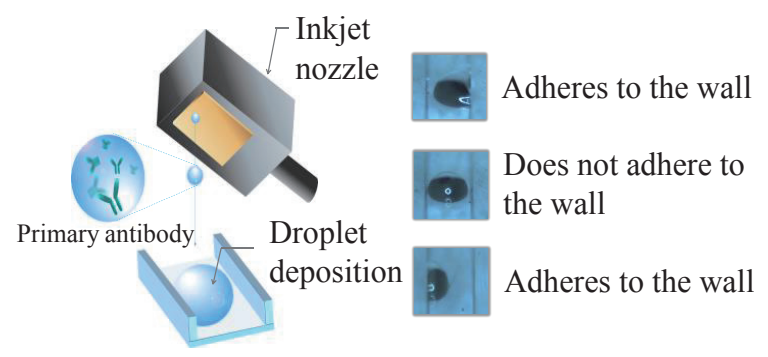

(b)

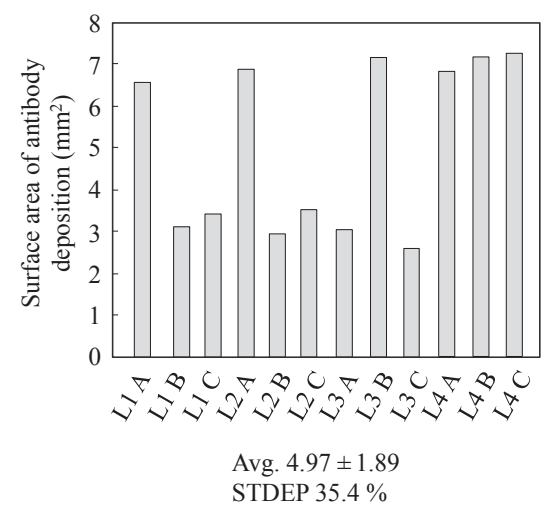

(c)

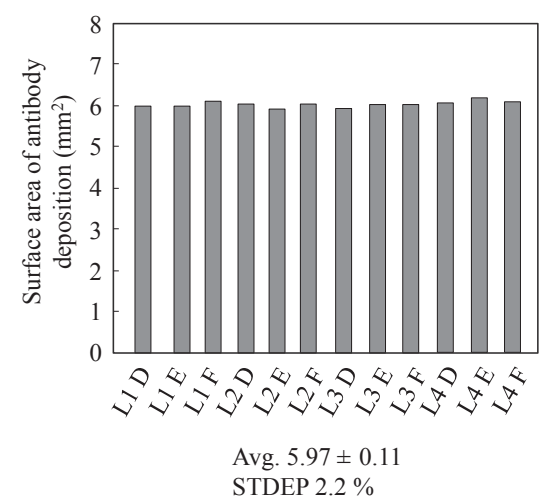

(d)

Fig. 2. (Color online) Schematic diagram for antibody deposition using a PulseInjector ${ }^{\circledR}$ and variation in the surface area of the deposited antibody spots: (a) PulseInjector ${ }^{\circledR}$ closely contacts the microchannel, (b) primary antibody deposition in the microchannel by the PulseInjector ${ }^{\circledR}$, (c) photo showing primary antibody spots on the microchannel without processing and comparison of the surface area for antibody deposition, and (d) photo showing primary antibody spots on the concave microchannel and comparison of the surface area for antibody deposition. Here, "processing" means generating the concave surface. 
replacement of reagents in the immunoassay process. This was addressed by forming concave surfaces of different depths $(0-20 \mu \mathrm{m})$ [Fig. 3(a)], then alternately introducing fluorescent and aqueous solutions into the microchannel [Fig. 3(b)]. The antibody was made fluorescent by modification with fluorescein isothiocyanate. The fluorescence intensity in the microchannel during replacement of the fluorescent solution with nonfluorescent solution was monitored using a fluorescence detector (FLE-1000, NSG Group, Tokyo, Japan). The excitation and emission wavelengths were 470 and $530 \mathrm{~nm}$, respectively. Figure 4 shows that significant fluorescence was detected when fluoresceinlabeled antibody solution filled the microchannel and that the intensity dropped when this solution was replaced by a non-fluorescent aqueous solution. Deeper channels provided correspondingly higher fluorescence intensity because the volume in the detection site increases in parallel. The fluorescence intensity of the aqueous solution remained essentially constant at concave area depths up to $10 \mu \mathrm{m}$ but increased significantly at depths of $15 \mu \mathrm{m}$ and more due to incomplete replacement of the fluorescein-antibodylabelled solution in the concave surface part of the microchannel. Therefore, a $10 \mu \mathrm{m}$ deep concave area was used in subsequent experiments.

\begin{tabular}{|l|l|l|l|l|l|}
\hline Depth of concave surface $(\mu \mathrm{m})$ & 0 & 5 & 10 & 15 & 20 \\
\hline Image & & & & & \\
\hline
\end{tabular}

(a)

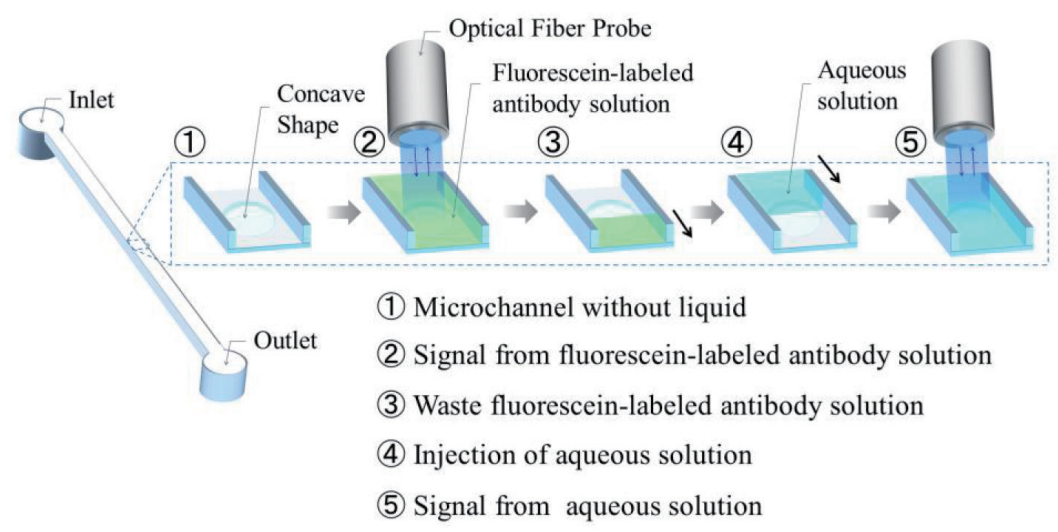

(b)

Fig. 3. (Color online) Method for investigating the complete replacement of reagent solutions in microchannels: (a) top view of concave surfaces $0,5,10,15$, and $20 \mu \mathrm{m}$ deep, and (b) procedure for measuring fluorescence intensity upon sequential replacement of the solution in the microchannel. 


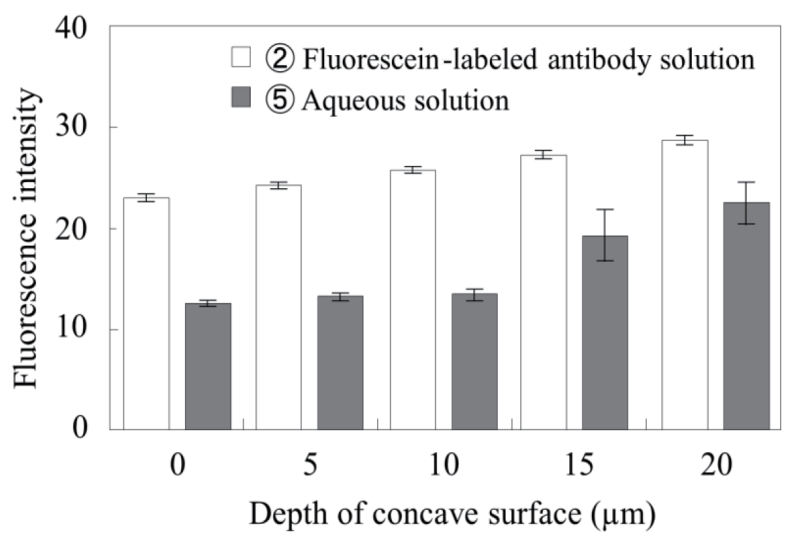

Fig. 4. Comparison of fluorescence intensity from fluorescein-labeled antibody solution ( $\square$ ); aqueous solution $(\square)$.

\subsection{Sandwich ELISA on a laser ablated concave undersurface in a microchannel}

In this study, PICP was used as a model analyte. The concentration of PICP in serum both correlates with the rate of bone formation ${ }^{(23)}$ and is used to estimate the rate of type I collagen synthesis in the body. Therefore, the serum concentration of PICP can be used as a biomarker for osteoporosis and osteoblastic bone metastases in prostate cancer.

We previously described that most antibodies tested could be immobilized on the PMMA microchannel surface following pulse laser ablation at $193 \mathrm{~nm}$ and $1.76 \mathrm{~J} \cdot \mathrm{cm}^{-2} \cdot{ }^{18,20)}$ A concave surface depth of $10 \mu \mathrm{m}$ supported uniform printing of antibody patterns (Fig. 2) and complete solution replacement in the microchannel (Fig. 4). The utility of this approach for a sandwich ELISA was further demonstrated by investigating different microchannel surfaces [Figs. 5(a) and 5(b)]. The printed surfaces of "concave shape" and "concave shape and laser ablation" in Fig. 5(a) are uniform in comparison to that of "without processing" and "laser ablation". Here, "processing" means generating the concave surface. The "concave shape" does not cause droplets to adhere to the wall, which is promising, but is insufficient for providing a uniformly printed surface. PICP imaged on a microchip is shown in Fig. 5(b) and reproducibility and RSDs are shown in Table 1. Essentially no luminescence intensity from 3 spots in the channel "without processing" and "concave shape" was detected, and the RSDs were 1.98 and $10.92 \%$, respectively, indicating that the primary antibody was not immobilized on the microchannel surface. On the other hand, significant luminescence for "laser ablation" and "concave shape and laser ablation" was observed, with RSDs of 8.07 and 1.94\%, respectively. Furthermore, "concave shape" provided slightly higher luminescence due to increased volume of the sample spot. Reproducibility studies showed low variation for "concave shape and laser ablation" because of the concave surface, indicating that "concave shape and laser ablation" provides the properties required for a capillary-flowdriven single channel immunoassay system. 


\begin{tabular}{|l|c|c|c|c|}
\hline & $\begin{array}{c}\text { Without } \\
\text { processing }\end{array}$ & $\begin{array}{c}\text { Concave } \\
\text { shape }\end{array}$ & $\begin{array}{c}\text { Laser } \\
\text { ablation }\end{array}$ & $\begin{array}{c}\text { Concave shape } \\
\text { \& laser ablation }\end{array}$ \\
\hline $\begin{array}{l}\text { Before } \\
\text { printing }\end{array}$ & & & & \\
\hline $\begin{array}{l}\text { After } \\
\text { printing }\end{array}$ & & & & \\
\hline
\end{tabular}

(a)

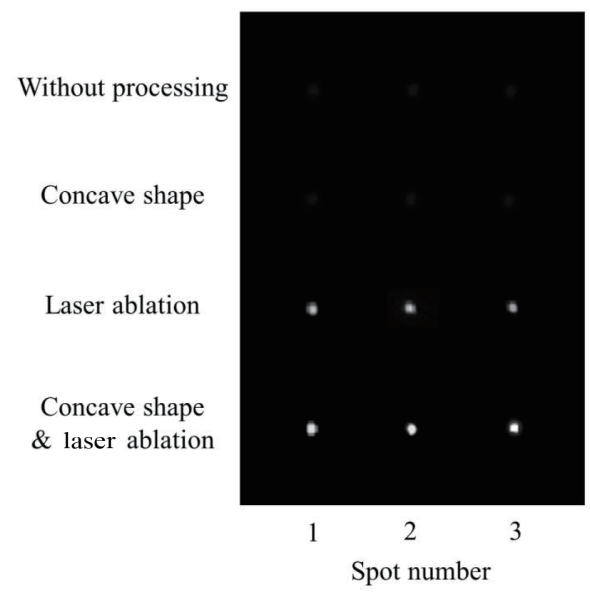

(b)

Fig. 5. Detection using a sandwich ELISA system with different types of microchannel surfaces: (a) top view a microchannel surface before and after printing, and (b) luminescence image of the corresponding microchannel surfaces.

Table 1

Reproducibility of fluorescence intensity of PICP against different types of microchannel surfaces.

\begin{tabular}{lcccc}
\hline Spot number & $\begin{array}{c}\text { Without } \\
\text { processing }\end{array}$ & $\begin{array}{c}\text { Concave } \\
\text { shape }\end{array}$ & $\begin{array}{c}\text { Laser } \\
\text { ablation }\end{array}$ & $\begin{array}{c}\text { Concave shape } \\
\text { and laser ablation }\end{array}$ \\
\hline 1 & 3517 & 3785 & 53617 & 62013 \\
2 & 3381 & 4786 & 57518 & 59182 \\
3 & 3535 & 3871 & 47188 & 60171 \\
Average & 3478 & 4147 & 52774 & 60455 \\
RSD (\%) & 1.98 & 10.92 & 8.07 & 1.94 \\
\hline
\end{tabular}




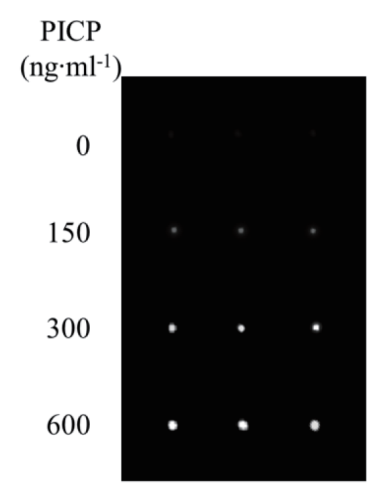

(a)

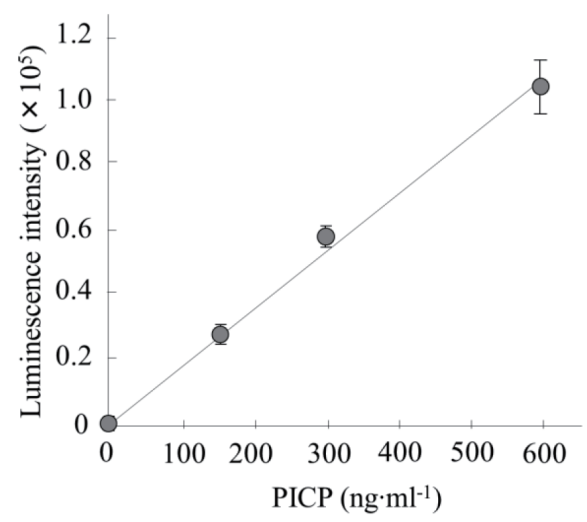

(b)

Fig. 6. Microfluidic chip responses and quantitative analyses of PICP concentration: (a) luminescence image of the microchip for $0,150,300$, and $600 \mathrm{ng} \cdot \mathrm{ml}^{-1}$, and (b) response curve for different PICP concentrations.

Thus far, the capillary-driven flow in microchannels has provided as simple and accurate a screening methodology as our previously reported microfluidic immunoassay system..$^{(9-11,18,20)}$ Basically, all experiments were performed by measuring the luminescence intensity of 3 spots: the reliability of these standard deviations was less than $10 \%$. Following this procedure, three spots of primary antibody were deposited onto separate concave surfaces in the microchannel, then the effects of microfluidic chip responses on PICP concentration were investigated in the range $0-600 \mathrm{ng} \cdot \mathrm{ml}^{-1}$. The luminescence intensity increased in a PICP concentration-dependent manner [Fig. 6(a)]. The mean luminescence intensity for 3 different spots at each PICP concentration plotted against concentration provided a linear relationship [Fig. 6(b)]. This range is adequate for clinical estimation of the PICP concentration in the blood. ${ }^{(24,25)}$ These data strongly indicate that this microchannel sandwich ELISA is suitable for the determination of PICP concentration.

\section{Conclusions}

Our goal was to develop a practical POCT microfluidic system based on capillarydriven flow. To this end, we described the formation of a concave surface on the undersurface of a microchannel using nanosecond pulse laser ablation to provide a surface amenable to antibody immobilization. A concave surface $10 \mu \mathrm{m}$ deep and 260 $\mu \mathrm{m}$ in diameter was demonstrated to be effective for uniform antibody printing onto the microchannel surface. A fluorescently-labeled antibody solution could be completely replaced with an aqueous solution using channel depths up to $10 \mu \mathrm{m}$. Concave microchannel surfaces $10 \mu \mathrm{m}$ deep and $260 \mu \mathrm{m}$ in diameter supported the sensitive and accurate determination of luminescence intensity based on a sandwich ELISA system. Furthermore, quantitative analysis of PICP concentrations showed a linear relationship in the range $0-600 \mathrm{ng} \cdot \mathrm{ml}^{-1} \mathrm{PICP}$. 


\section{Acknowledgements}

This work was supported by the Amada Foundation for Laser Processing and a Grantin-Aid for Scientific Research from the Ministry of Education, Culture, Sports, Science and Technology, Japan (24710146).

\section{References}

1 K. Sato, M. Tokeshi, H. Kimura and T. Kitamori: Anal. Chem. 73 (2001) 1213.

2 K. Sato, M. Yamanaka, H. Takahashi, M. Tokeshi, H. Kimura and T. Kitamori: Electrophoresis 23 (2002) 734.

3 K. Sato, M. Yamanaka, T. Hagino, M. Tokeshi, H. Kimura and T. Kitamori: Lab Chip 4 (2004) 570 .

4 H. Nagai, Y. Narita, M. Ohtaki, K. Saito and S. Wakida: Anal. Sci. 23 (2007) 975.

5 N. Matsunaga, S. Furutani and I. Kubo: ECS Trans. 16 (2008) 123.

6 K. Sato, K. Mawatari and T. Kitamori: Lab Chip 8 (2009) 1992.

7 I. Kubo, T. Kanamatsu and S. Furutani: Sens. Mater. 26 (2014) 615.

8 T. N. Stewart, B. E. Pierson, R. Aggarwal and R. J. Narayan: Biotechnol. J. 4 (2009) 206.

9 S. Yatsushiro, R. Akamine, S. Yamamura, M. Hino, K. Kajimoto, K. Abe, H. Abe, J. Kido, M. Tanaka, Y. Shinohara, Y. Baba, T. Ooie and M. Kataoka: PLoS One 6 (2011) e18807.

10 K. Abe, Y. Hashimoto, S. Yatsushiro, S. Yamamura, M. Bando, Y. Hiroshima, J. Kido, M. Tanaka, Y. Shinohara, T. Ooie, Y. Baba and M. Kataoka: PLoS One 8 (2013) e53620.

11 Y. Fuchiwaki, Y. Yabe, Y. Adachi, M. Tanaka, K. Abe, M. Kataoka and T. Ooie: Sens. Actuators, A 219 (2014) 1.

12 C. Wochnowski, M. A. Shams Eldin and S. Metev: Polym. Degrad. Stab. 89 (2005) 252.

13 X. L. Zhu, S. B. Liu, B. Y. Man, C. Q. Xie, D. P. Chen, D. Q. Wang, T. C. Ye and M. Liu: Appl. Surf. Sci. 253 (2007) 3122.

14 E. Stratakis, A. Ranella, M. Farsari and C. Fotakis: Prog. Quantum Electron. 33 (2009) 127.

15 L. He, J. Chen, D. F. Farson, J. J. Lannutti and S. I. Rokhlin: Appl. Surf. Sci. 257 (2011) 3547.

16 E. György, A. Pérez del Pino, J. Roqueta, C. Sánchez and A. G. Olivad: Colloids Surf., B: 104 (2013) 169.

17 P. Slepička, I. Michaljaničová, P. Sajdl, P. Fitl and V. Švorčík: Appl. Surf. Sci. 283 (2013) 438.

18 M. Tanaka, T. Ooie, Y. Yamachoshi, T. Nakahara, M. Hino, R. Akamine and M. Kataoka: J. Laser Micro/Nanoeng. 5 (2010) 35.

19 Q. Yu, X. Zhan, K. Liu, H. Lv and Y. Duan: Anal. Chem. 85 (2013) 4578.

20 Y. Yamachoshi, M. Tanaka, T. Nakahara, K. Abe, M. Kataoka and T. Ooie: J. Laser Micro/ Nanoeng. 7 (2012) 35.

21 D. Teixidor, T. Thepsonthi, J. Ciurana and T. Özel: J. Manuf. Processes 14 (2012) 435.

22 I. A. Paun, A. Selimis, G. Bounos, G. Kecskeméti and S. Georgiou: Appl. Surf. Sci. 255 (2009) 9856.

23 J. Ristell, J. Melkko, S. Niemi and L. Ristell: Calcif. Tissue Int. 49 (1991) S24.

24 A. M. Parfitt, L. S. Simon, A. R. Villanueva and S. M. Krane: J. Bone Miner. Res. 2 (1987) 427.

25 K. Kohda, N. Ito, M. Ohwada, K. Morita, N. Watanabe, Y. Kohgo, Y. Mogi and Y. Niitsu: Gut 32 (1991) 624. 